\title{
EDITORIAL
}

\section{Pulmonary dendritic cells: playing ball in the BAL?}

\author{
I.K. Demedts, G.F. Joos and G.G. Brusselle
}

$\mathbf{T}$ he lung is one of the few organs in the human body where there is a continuous and extensive interaction between the environment and our immune system. With every breath we take, numerous airborne particles, both harmless as well as potentially dangerous, are inhaled into the airways. The big challenge for the pulmonary immune system is to discriminate the good from the bad and to react accordingly. Unnecessary action against harmless particles (e.g. innocent antigens, self proteins) should be avoided, while a rapid and strong immune response is needed against potentially dangerous microorganisms. Pulmonary dendritic cells (DCs) are ideally suited to maintain this delicate balance between tolerance and active immune responses [1]. DCs are antigen-presenting cells that play a central role in host immune defence, by linking innate with adaptive immune responses [2]. They recognise danger signals from invading pathogens and are able to prime naïve T-cells and to initiate appropriate T-cell immune responses against these microorganisms [3] Indeed, T-lymphocytes themselves are actually "blind" to unprocessed antigens, and require the assistance of antigen presenting cells, such as DCs and macrophages, to be able to recognise antigens. Moreover, by the release of inflammatory cytokines, DCs influence the polarisation of the adaptive T-cell response into either a T-helper cell type (Th)1-, Th2- or a Tregulatory cell-direction [4]. This enables them not only to initiate active immune responses, but also to control and dampen these immune responses, as well as to avoid unwanted immune responses against self-antigens (autoimmunity).

Human pulmonary DCs have been described in human lung in bronchoalveolar lavage (BAL) fluid [5], small airway epithelium [6] and lung parenchyma [7]. Importantly, a role for pulmonary DC in the pathogenesis of highly prevalent lung diseases such as asthma [8] and chronic obstructive pulmonary disease [6] has been suggested. However, most data on the role of pulmonary DCs in respiratory diseases are obtained from animal studies, and few data are available on the presence of DCs in human lungs from patients suffering from lung disease. DCs are present at very low numbers in human lung, which makes the study of human lung DCs technically difficult, very time consuming and labour-intensive.

Dept of Respiratory Diseases, Ghent University Hospital, Ghent, Belgium.

STATEMENT OF INTEREST: None declared.

CORRESPONDENCE: I.K. Demedts, Dept of Respiratory Diseases, Ghent University Hospital 7K12IE, De Pintelaan 185, B-9000 Ghent, Belgium. Fax: 32 93322341. E-mail: M.DemedtsIngelk@ UGent.be
In the current issue of the European Respiratory Journal, LOMMATZSCH et al. [9] describe the presence of different DC subsets in the BAL fluid of patients with sarcoidosis, idiopathic pulmonary fibrosis (IPF) or pneumonia, and compare them with nonsmoking healthy controls. This study is remarkable for several reasons. By using advanced laboratory techniques (four-colour flow cytometry), the authors provide an accurate description of the different DC subsets: $\mathrm{CD}_{123}{ }^{+} \mathrm{MHCII}$ plasmacytoid DC (pDC) and $\mathrm{CD}_{11 \mathrm{c}^{+} \mathrm{MHCII}}{ }^{+}$myeloid $\mathrm{DC}$ (mDC). In the mDC subset, the authors further distinguish a $\mathrm{CD}^{+}{ }^{+}$and a CD1a- subset. As mentioned previously, DCs are present at very low numbers in human lung, and the use of multiple markers is needed for the correct identification of DCs and to avoid contamination of the DC population with other cell types (e.g. BAL macrophages). Importantly, the authors included a control group of nonsmoking healthy controls, which is not easily obtained in studies using invasive techniques, such as bronchoscopy and BAL. Finally, for the first time, the authors studied the presence of DCs in BAL of three different lung diseases (pneumonia, IPF and sarcoidosis). Until now, very few, if any, data were available on the possible role of human BAL DCs in the pathogenesis of these diseases.

The major findings of the study from LOMMATZSCH et al. [9] can be summarised as follows: in the BAL of patients with sarcoidosis, there is a significant increase of a particular subset of myeloid DC. This suggests a role for the CD1a- $\mathrm{mDC}$ in the pathogenesis of sarcoidosis, especially in the early stages of the disease. This is an intriguing finding, since activated T-cells in the BAL are essential for the inflammatory process in sarcoidosis [10]. As noted above, this T-cell mediated immune response can only be the consequence of the interaction of $\mathrm{T}$ lymphocytes with antigen presenting cells such as DCs.

LOMMATZSCH et al. [9] also studied BAL fluid of patients with pneumonia, both in immunocompetent and immunocompromised subjects. In immunocompetent patients with pneumonia, the number of $\mathrm{pDC}$ in the BAL was significantly higher than in control patients, while the relative number of $\mathrm{mDC}$ was lower. The authors state that the patients with pneumonia most likely suffered from pneumonia of bacterial origin. This again is surprising, since pDCs are traditionally described as mediators of antiviral immune response, while myeloid DCs in human lung are able to initiate immune responses against bacterial microorganisms [11]. It remains unclear if the observed increase in $\mathrm{pDC}$ is due to an unidentified viral cause of pneumonia, or can be attributed to a previously unknown function of $\mathrm{pDC}$ in the defence against bacterial respiratory pathogens.

In immunocompromised patients with pneumonia, both $\mathrm{pDC}$ and $\mathrm{mDC}$ numbers are lower than in controls. This probably 
can be explained by an overall immunosuppressive effect on all inflammatory cells, both from the innate and from the adaptive immune system.

Finally, in patients with IPF, there is no difference in BAL DC number when compared with healthy controls. This is consistent with the current knowledge on the pathogenesis of IPF, which is characterised mainly by fibrotic changes to the epithelium and the extracellular matrix, whereas only modest inflammation is observed [12].

The question now is: what do these findings mean and how can they be explained? Are the changes in BAL DC numbers just the result of "spill-over" of parenchymal inflammation into the BAL, or do they actually reflect a pivotal role for these DC in the pathogenesis of pulmonary diseases? If so, what is the interaction between DC and other inflammatory cell types in the BAL, such as macrophages, neutrophils and Tlymphocytes? What is the driving mechanism behind the changes in DC numbers in the BAL? While the study of LOMMATZSCH et al. [9] provides new and interesting information, it does not provide answers to these questions. Since a functional analysis of the DC subsets in these different lung diseases is lacking, the functional role of the different DC subsets in human BAL remains unclear. Moreover, the mechanisms responsible for the migration of DC towards the BAL are far from elucidated. There are no data on the expression of chemokine receptors, nor on the cytokine release by the different BAL DC subsets. From this point of view, the current study should be regarded as a pilot study, prompting further research on the function of BAL DC in health and disease.

In conclusion, LOMMATZSCH et al. [9] demonstrate, for the first time, important changes in BAL DC numbers in a variety of pulmonary diseases. As with every interesting new study, their work leaves us with several new and unanswered questions, the most important being: do dendritic cells actually play an active role in the BAL compartment? Do BAL DC indeed participate in the pathogenesis of these respiratory diseases or are the changes observed by LOMMATZSCH et al. [9] just "insignificant" epiphenomena of disease processes that are driven by mechanisms other than DC-initiated inflammatory responses?

The answer lies in a joined effort of pulmonary physicians, basic scientists and dendritic-cell biologists to initiate new studies (both in animal models and in human subjects), to further unravel the exact function of these enigmatic pulmonary dendritic cells. So let's play ball!

\section{REFERENCES}

1 Vermaelen K, Pauwels R. Pulmonary dendritic cells. Am J Respir Crit Care Med 2005; 172: 530-551.

2 Banchereau J, Steinman RM. Dendritic cells and the control of immunity. Nature 1998; 392: 245-252.

3 Guermonprez P, Valladeau J, Zitvogel L, Théry C, Amigorena S. Antigen presentation and T cell stimulation by dendritic cells. Annu Rev Immunol 2002; 20: 621-667.

4 Kapsenberg ML. Dendritic-cell control of pathogen-driven T-cell polarization. Nat Rev Immunol 2003; 3: 984-993.

5 van Haarst JM, Hoogsteden HC, de Wit HJ, Verhoeven GT, Havenith CE, Drexhage HA. Dendritic cells and their precursors isolated from human bronchoalveolar lavage: immunocytologic and functional properties. Am J Respir Cell Mol Biol 1994; 11: 344-350.

6 Demedts IK, Bracke KR, Van Pottelberge G, et al. Accumulation of dendritic cells and increased CCL20 levels in the airways of patients with chronic obstructive pulmonary disease. Am J Respir Crit Care Med 2007; 175: 998-1005.

7 Demedts IK, Brusselle GG, Vermaelen KY, Pauwels RA. Identification and characterization of human pulmonary dendritic cells. Am J Respir Cell Mol Biol 2005; 32: 177-184.

8 Lambrecht BN, Hammad H. Taking our breath away: dendritic cells in the pathogenesis of asthma. Nat Rev Immunol 2003; 3: 994-1003.

9 Lommatzsch M, Bratke K, Bier A, et al. Airway dendritic cell phenotypes in inflammatory diseases of the human lung. Eur Respir J 2007; 30: 878-886.

10 Grunewald J, Eklund A. State of the art. Role of CD4+ T cells in sarcoidosis. Proc Am Thorac Soc 2007; 4: 461-464.

11 Demedts IK, Bracke KR, Maes T, Joos GF, Brusselle GG. Different roles for human lung dendritic cell subsets in pulmonary immune defense mechanisms. Am J Respir Cell Mol Biol 2006; 35: 387-393.

12 Hunninghake GW, Schwarz MI. State of the art. Does current knowledge explain the pathogenesis of idiopathic pulmonary fibrosis?: a perspective. Proc Am Thorac Soc 2007; 4: 449-452. 